\title{
Association between renal function and retinal neurodegeneration in Chinese patients with type 2 diabetes mellitus
}

\author{
Xia Gong ${ }^{1}$, Wei Wang ${ }^{1}$, Wangting $\mathrm{Li}^{1}$, Ling Jin ${ }^{1}$, Lanhua Wang ${ }^{1}$, Jie Meng ${ }^{1}$, Kun Xiong ${ }^{1}$, Yuting Li $^{1}$, \\ Xiao Han ${ }^{2}$, Xiaoling Liang ${ }^{1}$, Wenyong Huang ${ }^{1}$ \\ ${ }^{1}$ Zhongshan Ophthalmic Center, State Key Laboratory of Ophthalmology, Sun Yat-sen University, Guangzhou, China; ${ }^{2}$ The Seventh Affiliated \\ Hospital, Sun Yat-sen University, Shenzhen, China \\ Contributions: (I) Conception and design: W Huang, W Wang; (II) Administrative support: Y Li; (III) Provision of study materials or patients: \\ W Huang, X Liang; (IV) Collection and assembly of data: X Gong, W Li, W Wang, L Wang, J Meng, K Xiong, X Han; (V) Data analysis and \\ interpretation: X Gong, W Wang, L Jin; (VI) Manuscript writing: All authors; (VII) Final approval of manuscript: All authors. \\ Correspondence to: Wenyong Huang, MD, PhD. Professor of Ophthalmology, Zhongshan Ophthalmic Center, State Key Laboratory of \\ Ophthalmology, Sun Yat-sen University, 54S. Xianlie Road, Guangzhou 510060, China. Email: hweny@mail.sysu.edu.cn.
}

Background: Chronic kidney disease (CKD) and diabetic retinopathy (DR) are two serious complications of diabetes. However, the association between retinal neurodegeneration in DR and renal function decline is still unclear. Our objective was to evaluate the association by measure estimated glomerular filtration rate (eGFR), macular ganglion cell-inner plexiform layer (GC-IPL), and ganglion cell complex (GCC) thickness in patients with type 2 diabetes mellitus (T2DM).

Methods: We analyzed the baseline data of the Guangzhou Diabetic Eye Study. T2DM patients from communities in Guangzhou were enrolled and all participants went through ophthalmic and general examinations. The thickness of the macular GC-IPL and GCC in their right eyes were measured by sweptsource optical coherence tomography. CKD was defined as eGFR $<60 \mathrm{~mL} / \mathrm{min} / 1.73 \mathrm{~m}^{2}$.

Results: One thousand three hundred and nine patients were included (mean age $64.4 \pm 7.6$ years, 59.1\% female), and fifty-eight (4.4\%) of them had CKD. Average macular GC-IPL thickness was significantly thinner in CKD patients $(96.5 \pm 9.1 \mu \mathrm{m})$ than non-CKD patients $(101.3 \pm 9.2 \mu \mathrm{m})(\mathrm{P}<0.01)$. Average macular GCC thickness was also significantly thinner in CKD patients $(123.5 \pm 13.2 \mu \mathrm{m})$ than non-CKD patients $(129.9 \pm 12.8 \mu \mathrm{m})(\mathrm{P}<0.01)$. The significant thinning of macular GC-IPL and GCC thickness presented in every gird in the macula (all, $\mathrm{P}<0.05$ ) except for central grid $(\mathrm{P} \geq 0.05)$. In the patients without $\mathrm{DR}$, the eGFR was linearly correlated with the average macular GC-IPL thickness [ $\beta=0.07$ (95\% CI, 0.02-0.12), $\mathrm{P}<0.01]$ and GCC thickness [ $\beta=0.09$ (95\% CI, 0.03-0.16), $\mathrm{P}<0.01]$ after adjustment for age, sex, axial length, intraocular pressure (IOP) and combination of hypertension. However, no linear correlation was found between eGFR and macular GC-IPL or GCC thickness in DR patients.

Conclusions: Renal function decrease is associated with the thinning of the macular GC-IPL and GCC in T2DM patients, suggesting the potential value of ganglion cell lose to detect early function decline in the kidney in diabetic patients, especially in patients without DR.

Keywords: Diabetes; neurodegeneration; optical coherent tomography; renal function

Submitted Oct 16, 2020. Accepted for publication Jan 15, 2021.

doi: 10.21037/atm-20-6957

View this article at: http://dx.doi.org/10.21037/atm-20-6957 


\section{Introduction}

Chronic kidney disease (CKD) is one of the major complications in diabetic patients. As CKD develops, not only will it progress into renal failure, but it is also an independent risk factor for cardiovascular disease, cognitive dysfunction, and all-cause mortality. With the enlarge of diabetic population, the number of patients with CKD has increased dramatically in recent decades and has become one of the greatest global health challenges of our time $(1,2)$. However, most CKD patients were diagnosed after renal function obvious decline. Thus, it is important to detect renal impairment in the early stage of CKD development (2).

Diabetic retinopathy (DR) is another major complications in diabetic patients (3). In many countries, DR, which develops in approximately $20-40 \%$ of diabetic patients (4), is the most common cause of avoidable blindness in the working-age population (5). Diabetic patients are suggested to take regular screening for DR.

Because both CKD and DR are microvascular complications of diabetes, researchers suggested that CKD and DR may be closely linked (6). The earliest clinical feature of DR is vascular changes, while retinal ganglion cell-inner plexiform layer (GC-IPL) thickness and ganglion cell complex (GCC) thickness are known to be early indicators of hyperglycemia-induced neurodegeneration prior to the detectability of clinical microvascular abnormalities (7-9). Hyperglycemia-induced neurodegeneration affects primarily the retinal ganglion cell nuclei and dendrites. This then affects the axons of the ganglion cell, and this is manifested as the diffuse thinning of the retinal GC-IPL followed by the thinning of the retinal nerve fiber layer (RNFL) (7). Since GCC is the combination of GC-IPL and RNFL, it will become thinner in this process. Moreover, researchers suggested that neurodegeneration in retina may associated with renal function decline in diabetic patients (10).

However, the association of the important parameters of neurodegeneration in DR development, including the thickness of the macular GC-IPL and GCC, with the eGFR (the most valuable indicator of overall renal function) (11), has not been elucidated. To fill this gap, we analyzed the baseline data of the Guangzhou Diabetic Eye Study to evaluate the association. This study is the first to use swept-source optical coherence tomography (SS-OCT) to investigate the association between retinal ganglion cell loss and renal function in T2DM patients in China.
We present the following article/case in accordance with the Strengthening the Reporting of Observational Studies in Epidemiology (STROBE) reporting checklist (available at http://dx.doi.org/10.21037/atm-20-6957).

\section{Methods}

\section{Subjects}

The Guangzhou Diabetic Eye Study is a prospective study conducted in the Zhongshan Ophthalmic Center, which is affiliated with Sun Yat-Sen University in Guangzhou, China. The study was conducted in accordance with the Declaration of Helsinki (as revised in 2013). The study was approved by the Zhongshan Ophthalmic Center Ethics Committee (No.: 2017KYPJ094) and informed consent was taken from all individual participants. This article analyzed its baseline data collected from October 2017 to April 2019.

The study recruited 1,473 T2DM patients between the ages of 30 and 80 . They were already registered in the health care systems in the communities near the Zhongshan Ophthalmic Center. Patients with any evidence of the following conditions were excluded: (I) best corrected visual acuity (BCVA) worse than 20/200, axial length $>30 \mathrm{~mm}$ or unmeasurable, spherical equivalent $(\mathrm{SphE}) \leq-12.0$ degrees, astigmatism $>4$ degrees, or intraocular pressure (IOP) $>21 \mathrm{mmHg}$ in the right eye; (II) except DR, other combined eye diseases that could affect retinal thickness in the right eye, such as glaucoma, age-related macular degeneration, and retinal detachment; (III) surgery or invasive treatment or laser treatment history on the right eye; (IV) severe systemic diseases, such as uncontrolled hypertension, severe cardiovascular and cerebrovascular disease, malignant tumors, and nephritis; (V) general surgery history, such as heart bypass, thrombolysis, and kidney transplantation; (VI) cognitive disorders or mental illness that would hinder the patient's cooperation with tests; and (VII) inability to obtain clear fundus or SS-OCT images because of refractive media opacity or noncooperation.

We excluded 40, 47, 77 patients for unqualified general condition, eye condition or image quality, respectively. One thousand three hundred and nine diabetic patients were included.

\section{General information and laboratory measurements}

Upon enrollment in the study, general information, including age, sex, diabetes duration, other combined 
systemic chronic diseases, medication compliance, were collected via questionnaires.

Height and weight were manually measured. Body mass index (BMI) was defined as weight $(\mathrm{kg})$ divided by the square of height $(\mathrm{m})$, and a patient with a BMI of 23-27.5 or $>27.5 \mathrm{~kg} / \mathrm{m}^{2}$ was considered to be overweight or obese, respectively (12).

Each patient's systolic blood pressure (SBP) and diastolic blood pressure (DBP) were measured by electronic sphygmomanometer (Hem-907, Omron, Kyoto, Japan). The mean artery pressure (MAP) was calculated to be the sum of $1 / 3$ SBP and 2/3 DBP.

Blood and urine samples were collected by experienced nurses. Serum creatinine, hemoglobin A1c (HbA1c), total cholesterol (TC), high-density lipoprotein cholesterol (HDL-C), low-density lipoprotein cholesterol (LDL-C), triglycerides (TG), C-reactive protein, and microalbuminuria were measured. The eGFR was calculated with the Xiangya formula, which has been reported to be the most accurate eGFR formula for the Chinese population (13). CKD was defined as an eGFR lower than $60 / \mathrm{min} / 1.73 \mathrm{~m}^{2}$ (14).

The hypertension was defined as: (I) $\mathrm{SBP} \geq 140 \mathrm{mmHg}$ and/or DBP $\geq 90 \mathrm{mmHg}$ at least three times in different days, and further diagnosed by physicians; (II) a history of hypertension or hypotensive drugs. Hyperlipidemia was defined as: (I) have one or a combination of the following: high HDL-C levels, high LDL-C levels, high total cholesterol levels, high triglycerides levels; (II) a history of hyperlipidemia or lipid-lowering drugs.

\section{Eye examination}

Each participant underwent a comprehensive ocular examination that included the best correct visual acuity (BCVA) test [Early Treatment Diabetic Retinopathy Study (ETDRS) LogMAR visual acuity chart, Precision Vision, Artesia, USA], refraction powers examination (auto refractometer, KR-8800, Topcon, Tokyo, Japan), IOP measurement (non-contact tonometer, CT-1, Topcon, Tokyo, Japan), slit lamp examination, and ocular biometric measurements (Lenstar LS900, Haag-Streit, Koeniz, Switzerland). The SphE was calculated as the sphere degree plus $1 / 2$ cylinder degree. The standard ETDRS 7-field fundus photography (CR-2, Canon, Tokyo, Japan) was obtained after the pupil was dilated with 3 drops of tropicamide phenylephrine (Mydorin, Santen, Osaka,
Japan). The images were graded by two well-trained graders in accordance with the English National Health Service Diabetic Eye Screening Programme Grading Classification Standard (15).

\section{Swept-source optical coberence tomography}

A SS-OCT device (DRI OCT Triton, Topcon, Tokyo, Japan) was used to scan the fovea area. A three-dimensional (3D) imaging scan protocol of native software (10.15.003.01) was used to evaluate a $6 \times 6 \mathrm{~mm}$ region of the macula. The scans were centered automatically and confirmed by a fundus camera that was integrated into the instrument. In accordance with the ETDRS standard, three circles with diameters of 1, 3, $6 \mathrm{~mm}$ divided the macula into a central grid, an inner ring and an outer ring; then inner ring and outer ring were divided into four quadrants, including superior, inferior, nasal and temporal quadrants. The thickness profiles of the GC-IPL and GCC layers were automatically produced, and thickness of GC-IPL or GCC in each grid was automatically reported.

The examinations were performed by an experienced examiner with no knowledge of the study protocol. Each participant's measurements were obtained through dilated pupil. The exclusion of unqualified images, including out-of-focus images or those with motion artifacts or segmentation algorithm failures, was performed at a different time from that of image acquisition.

\section{Statistical analysis}

The data from each participant's right eye was analyzed. The difference in characteristics between the CKD patients and non-CKD patients was evaluated through the independent samples $t$-test for the normally distributed data, Spearman's rank-sum test for the non-normally distributed data, and chi-square test for the qualitative data respectively. Univariate and multivariate linear regressions were used to evaluate the relationships between the eGFR and macular GC-IPL and GCC thickness. Model 1 of the multivariable linear regression was adjusted for the factors known to be relevant to macular GC-IPL and GCC thickness. These factors included age, sex, and axial length (16). Model 2 was further adjusted for the parameters that were associated with macular GC-IPL and GCC thickness in the univariable linear regression. These factors included hypertension and IOP. A P value of $<0.05$ was considered significant. 


\section{Results}

A total of 1,309 diabetic patients (59.1\% female) were included in the final analysis. The demographic and clinical characteristics are presented in Table 1. They had an average age of $64.4 \pm 7.6$ years [mean \pm standard deviation $(\mathrm{SD})]$. The median (interquartile range) diabetic duration was 7.0 (3.0$13.0)$ years. The proportions of overweight (51.6\%), obesity (16.8\%), hypertension $(56.5 \%)$, or hyperlipidemia (47.2\%) were high. Participants had a vision of average $\operatorname{LogMAR}$ BCVA (SD) 0.2 (0.1). Among the participants, 1,094 (83.6\%) had no signs of DR and 215 (16.4\%) had DR.

Fifty-eight (4.4\%) participants had CKD. Their average (SD) eGFR was $54.2(5.2) \mathrm{mL} / \mathrm{min} / 1.73 \mathrm{~m}^{2}$. Forty-six of them had an eGFR $\geq 50 \mathrm{~mL} / \mathrm{min} / 1.73 \mathrm{~m}^{2}$. Only 1 patient had an eGFR $<40 \mathrm{~mL} / \mathrm{min} / 1.73 \mathrm{~m}^{2}\left(36.8 \mathrm{~mL} / \mathrm{min} / 1.73 \mathrm{~m}^{2}\right)$. CKD patients were older than non-CKD patients $(70.7 \pm 6.6$ years for CKD, $64.1 \pm 7.5$ years for non-CKD, $\mathrm{P}<0.01)$. In addition, $\mathrm{CKD}$ patients had longer diabetes duration, higher BMI, higher C-reactive protein, higher microalbuminuria, worse BCVA, and, unexpectedly, lower IOP. The CKD patients were more likely to have hypertension, hyperlipidemia or have a requirement for insulin treatment (all $\mathrm{P}<0.05)$.

CKD presented in $13 / 215$ (6.0\%) DR patients and 45/1,049 (4.3\%) non-DR patients. The CKD proportion was higher in DR patients than non-DR patients, however there was no significant difference between two groups in CKD proportion (chi-square test, $\mathrm{P}=0.21$ ). Similarly, in CKD patients, the average (SD) eGFR was higher in patients without DR $\left(55.0 \pm 4.4 \mathrm{~mL} / \mathrm{min} / 1.73 \mathrm{~m}^{2}\right)$ than with DR $\left(51.9 \pm 7.0 \mathrm{~mL} / \mathrm{min} / 1.73 \mathrm{~m}^{2}\right)$, but the difference was not statistically significant $(\mathrm{P}=0.06)$.

We compared within the group of DR patients the features of patients with and without CKD. Besides measurements of renal function, significant differences were found in age $(67.9 \pm 9.1$ year for CKD, $63.2 \pm 7.7$ year for non-CKD, $\mathrm{P}=0.04$ ) and combination of hypertension (84.6\% for CKD, $54.5 \%$ for non-CKD, $\mathrm{P}=0.03$ ).

Table 2 shows the macular GC-IPL and GCC thickness in CKD patients and non-CKD patients. The average macular GC-IPL was thinner in CKD patients than nonCKD patients, with measurements of $101.3 \pm 9.2 \mu \mathrm{m}$ for nonCKD, $96.5 \pm 9.1 \mu \mathrm{m}$ for $\mathrm{CKD}$, respectively $(\mathrm{P}<0.01)$. The average macular GCC was also thinner in CKD patient than non-CKD patients, with measurements of $129.9 \pm 12.8 \mu \mathrm{m}$ for non-CKD, $123.5 \pm 13.2 \mu \mathrm{m}$ for CKD, respectively $(\mathrm{P}<0.01)$. The macular GC-IPL and GCC in the CKD patients were significantly thinner than non-CKD patients in almost every grid (all, $\mathrm{P}<0.05$ ), except for central grid $(\mathrm{P} \geq 0.05)$.

Table 3 presents the results of the univariate linear regression analysis between participants' characteristics and thickness of macular GC-IPL and GCC. Not only the eGFR, but also age, sex, axial length, IOP, and combination of hypertension or DR were significantly linearly correlated with macular GC-IPL or GCC thickness (all, $\mathrm{P}<0.05$ ).

Table 4 shows the results of the multivariate linear regression. To reduce the effect of the presence of DR, participants with and without DR were analyzed separately. Model 1 was adjusted for the factors known to be relevant to macular GC-IPL and GCC thickness. These included age, sex, and axial length. Model 2 further adjusted for the parameters associated with macular GC-IPL and GCC thickness in the univariate model. In the patients without DR, the linear correlation between the eGFR and GC-IPL and GCC persisted in all the models (all, $\mathrm{P}<0.01$ ). In model 2, eGFR was linearly correlated with the macular GC-IPL $[\beta=0.07$ (95\% CI, 0.02-0.012), $\mathrm{P}<0.01]$ and GCC thickness $[\beta=0.09$ (95\% CI, 0.03-0.016), $\mathrm{P}<0.01]$. However, in the DR patients, there was no significant linear correlation between eGFR and macular GC-IPL or GCC thickness (all, $\mathrm{P} \geq 0.05$ ).

\section{Discussion}

We have two main results: (I) the average macular GC-IPL and GCC thickness decreased in the CKD patients; (II) the eGFR was positively correlated with the average macular GC-IPL and GCC thickness independent of age, sex, axial length, and other potential confounding factors in patients without DR. Our results show a close association between retinal neurodegeneration and renal dysfunction and suggests that neurodegeneration progressed as renal function deteriorated. Thus, the potential for macular GC-IPL and GCC thickness to be indicative of early hyperglycemia-induced impairment in the eye and kidney in T2DM patients was supported.

We also have a few negative results. First, the macular GC-IPL and GCC thinning in CKD patients was found in every grid except the central grid. This could have been caused by the lower density of the ganglion cells in the fovea pit (17). Second, no significant linear correlation was found between macular GC-IPL and GCC thickness and the eGFR in the DR patients. One possible reason is the 
Table 1 Participant's characteristics stratified by the presence of chronic kidney diseases

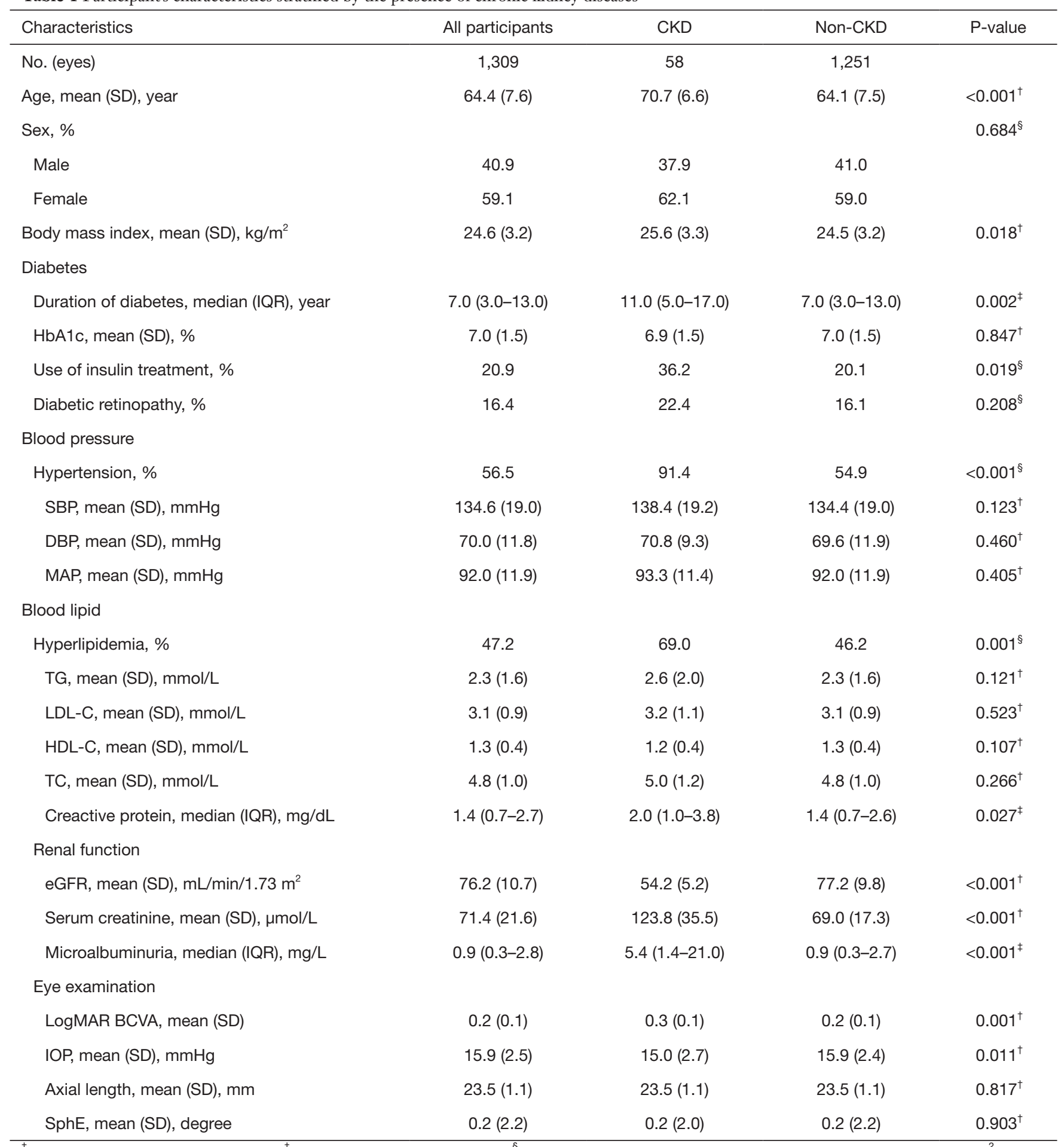

${ }^{\dagger}$, independent two-sample $t$-test; ${ }^{\ddagger}$, Spearman's rank-sum test; ${ }^{\S}$, Chi-square test. CKD defined as eGFR <60 mL/min/1.73 m². CKD, chronic kidney diseases; SD, standard deviation; IQR, interquartile range; SBP, systolic blood pressure; DBP, diastolic pressure; MAP, mean artery pressure; TG, triglyceride; LDL-C, low density lipoprotein cholesterol; HDL-C, high density lipoprotein cholesterol; TC, total cholesterol; eGFR, estimated glomerular filtration rate; BCVA, best corrected visual acuity; IOP, intraocular pressure; SphE, spherical equivalent. 
Table 2 Thickness of macular ganglion cell-inner plexiform layer and ganglion cell complex stratified by the presence of chronic kidney diseases

\begin{tabular}{|c|c|c|c|}
\hline Parameters & CKD $(\mu \mathrm{m})$ & Non-CKD $(\mu \mathrm{m})$ & $P$ value ${ }^{\dagger}$ \\
\hline $\begin{array}{l}\text { Macular GC-IPL } \\
\text { thickness }\end{array}$ & $96.5(9.1)$ & $101.3(9.2)$ & $<0.001$ \\
\hline Central grid & $43.2(14.0)$ & $45.5(18.0)$ & 0.339 \\
\hline Inner nasal grid & $103.6(13.2)$ & $109.8(13.2)$ & 0.001 \\
\hline Inner superior grid & $112.4(11.7)$ & 116.5 (12.2) & 0.011 \\
\hline Inner temporal grid & 100.9 (10.9) & $104.7(11.5)$ & 0.012 \\
\hline Inner inferior grid & $108.0(14.6)$ & $114.0(13.4)$ & 0.003 \\
\hline Average inner ring & $106.2(10.4)$ & $111.3(10.9)$ & 0.001 \\
\hline Outer nasal grid & $113.3(14.8)$ & $119.0(11.4)$ & 0.006 \\
\hline Outer superior grid & $101.2(12.5)$ & $106.0(12.2)$ & 0.006 \\
\hline Outer temporal grid & $88.3(8.5)$ & $92.3(10.4)$ & 0.001 \\
\hline Outer inferior grid & $97.1(13.6)$ & $103.5(11.1)$ & 0.001 \\
\hline Average outer ring & $100.0(10.6)$ & $105.2(9.8)$ & 0.001 \\
\hline $\begin{array}{l}\text { Macular GCC } \\
\text { thickness }\end{array}$ & 123.5 (13.2) & $129.9(12.8)$ & 0.001 \\
\hline Central grid & $47.3(18.6)$ & $49.8(22.5)$ & 0.414 \\
\hline Inner nasal grid & $125.8(17.4)$ & $133.2(17.0)$ & 0.002 \\
\hline Inner superior grid & 139.6 (15.2) & $145.0(16.6)$ & 0.012 \\
\hline Inner temporal grid & $120.5(14.1)$ & $124.9(14.9)$ & 0.025 \\
\hline Inner inferior grid & 133.5 (19.8) & $141.2(17.9)$ & 0.005 \\
\hline Average inner ring & $129.8(13.6)$ & $136.1(14.0)$ & 0.001 \\
\hline Outer nasal grid & $158.4(24.5)$ & $166.6(18.0)$ & 0.014 \\
\hline Outer superior grid & $141.0(19.7)$ & $147.5(18.9)$ & 0.018 \\
\hline Outer temporal grid & $110.2(11.6)$ & $115.5(14.0)$ & 0.001 \\
\hline Outer inferior grid & $135.3(22.5)$ & $145.0(20.8)$ & 0.002 \\
\hline Average outer ring & $136.2(16.5)$ & $143.7(14.9)$ & 0.001 \\
\hline
\end{tabular}

CKD defined as eGFR $<60 \mathrm{~mL} / \mathrm{min} / 1.73 \mathrm{~m}^{2}{ }^{\dagger}$, independent twosample $t$-test. CKD, chronic kidney diseases; SD, standard deviation; GC-IPL, ganglion cell-inner plexiform layer; GCC, ganglion cell complex.

relatively small sample size [215] of DR patients. Another is that the increase in retinal thickness in DR patients might have obscured the GC-IPL and GCC thinning caused by the loss of ganglion cells. In the progression of DR, retinal capillary leakage would lead to retinal edema (18). Moreover, it has been hypothesized that ganglion cell degeneration could play a role in the blood-retinal barrier breakdown which could induce retinal edema in the development of DR (19). The results of the linear regressions indicate that the macular GC-IPL and GCC in the DR patients tended to be thicker than patients without DR. Before diabetic macular edema becomes clinically significant, morphologic changes might not be observable in OCT images. It is assumed that the evaluation of neurodegeneration through OCT might be more valuable for patients without DR.

The retinal and renal glomerular microvasculature have many similarities, and diseases of these organs have a common pathogenesis. However, few studies have evaluated the association between neurodegeneration and renal function in diabetic patients. Srivastav et al. reported that an increase in serum creatinine levels was associated with RNFL thinning in diabetic patients (10). However, the study included only 60 diabetic patients and considered few relative factors. Moreover, serum creatinine has limited value as a single indicator of renal function because more than half of the patients with an eGFR of $<60 \mathrm{~mL} / \mathrm{min} / 1.73 \mathrm{~m}^{2}$ might have normal serum creatinine levels (14). The present study enrolled a large sample of T2DM patients in local communities and adjusted for multiple potential confounding factors. Thus, renal function was confirmed to be independently correlated with retinal neurodegeneration in diabetic patients.

There are also limitations. First, as previously mentioned, the use of macular GC-IPL and GCC thickness to evaluate ganglion cell loss might be confounded by the presence of DR. Because of the uneven distribution of DR patients, further studies are necessary to investigate retinal neurodegeneration and its association with renal function in patients with various severity levels of DR. Second, we only analyzed the baseline data; thus, it is also necessary to further investigate the synchronization of retinal neurodegeneration and renal function decline. Follow-up visits are ongoing. Third, this is not a population-based or hospital-based study. The participants were recruited from communities voluntarily. A majority did not have DR or CKD; thus, the generalization of the findings to other diabetic patients should be considered with caution. Most patients with diabetes in China are located in communities, thereby this study still can represent a large population.

\section{Conclusions}

In summary, we found that the progression of retinal 
Table 3 Univariate linear regression analysis of the relationship between characteristics and thickness of macular ganglion cell-inner plexiform layer or ganglion cell complex

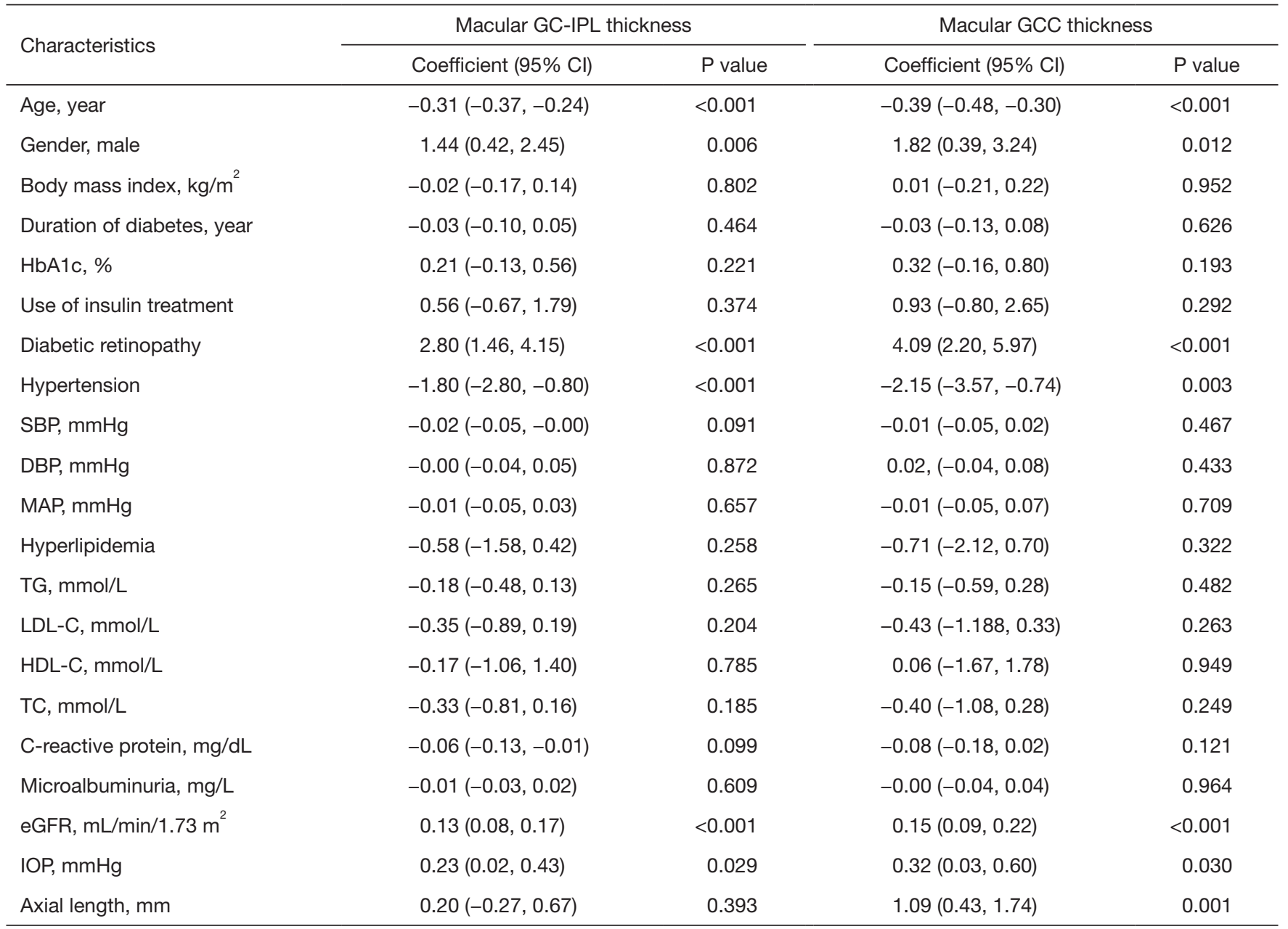

GC-IPL, ganglion cell-inner plexiform layer; GCC, ganglion cell complex; 95\% Cl, 95\% confidential interval; SD, standard deviation; SBP, systolic blood pressure; DBP, diastolic pressure; MAP, mean artery pressure; TG, triglyceride; LDL-C, low density lipoprotein cholesterol; HDL-C, high density lipoprotein cholesterol; TC, total cholesterol; IQR, interquartile range; eGFR, estimated glomerular filtration rate; IOP, intraocular pressure.

Table 4 Multivariate linear regression analysis of the relationship between estimated glomerular filtration rate (eGFR) and thickness of macular ganglion cell-inner plexiform layer or ganglion cell complex

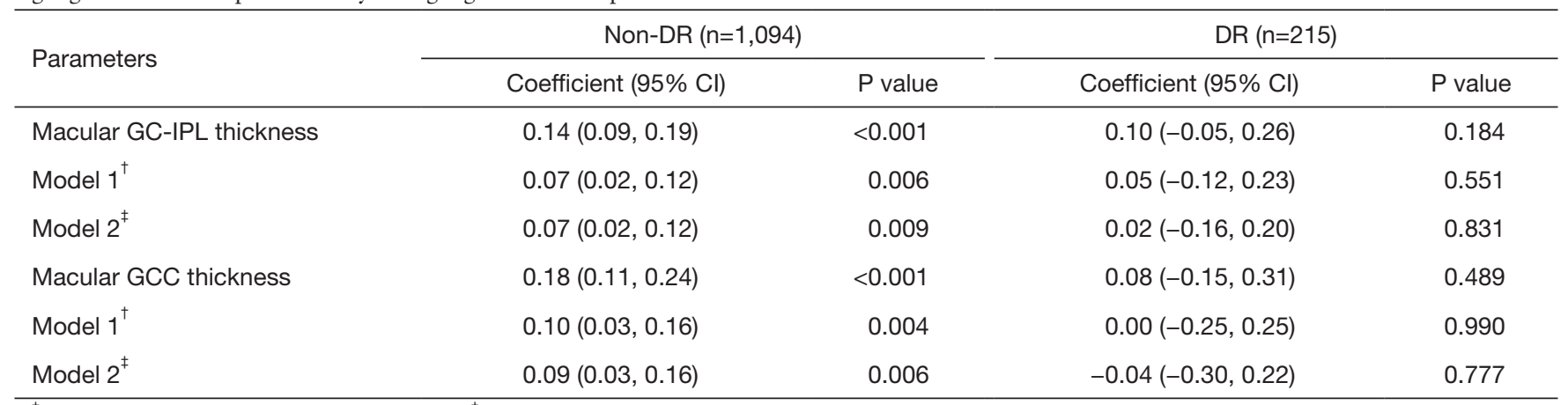

${ }^{\dagger}$, adjusted for age, gender, axial length; ${ }^{\ddagger}$, further adjusted for hypertension (yes =1; no =0), intraocular pressure. DR, diabetic retinopathy; 95\% Cl, 95\% confidential interval; GC-IPL, ganglion cell-inner plexiform layer; GCC, ganglion cell complex. 
neurodegeneration associated with the deterioration of renal function in Chinese T2DM patients. OCT is a noninvasive tool which can quantitatively evaluate GC-IPL and GCC thicknesses, but the evaluation is more valuable for patients without DR.

\section{Acknowledgments}

Funding: This work was supported by the National Natural Science Foundation of China (grand numbers 81570843; 81530028; 81721003), the Guangdong Province Science \& Technology Plan (grant number 2014B020228002).

\section{Footnote}

Reporting Checklist: The authors have completed the STROBE reporting checklist. Available at http://dx.doi. org/10.21037/atm-20-6957

Data Sharing Statement: Available at http://dx.doi. org/10.21037/atm-20-6957

Conflicts of Interest: All authors have completed the ICMJE uniform disclosure form (available at http://dx.doi. org/10.21037/atm-20-6957). The authors have no conflicts of interest to declare.

Ethical Statement: The authors are accountable for all aspects of the work in ensuring that questions related to the accuracy or integrity of any part of the work are appropriately investigated and resolved. The study was conducted in accordance with the Declaration of Helsinki (as revised in 2013). The study was approved by the Zhongshan Ophthalmic Center Ethics Committee (No.: 2017KYPJ094) and informed consent was taken from all individual participants.

Open Access Statement: This is an Open Access article distributed in accordance with the Creative Commons Attribution-NonCommercial-NoDerivs 4.0 International License (CC BY-NC-ND 4.0), which permits the noncommercial replication and distribution of the article with the strict proviso that no changes or edits are made and the original work is properly cited (including links to both the formal publication through the relevant DOI and the license). See: https://creativecommons.org/licenses/by-nc-nd/4.0/.

\section{References}

1. Drawz P, Rahman M. Chronic Kidney Disease. Ann Intern Med 2015;162:ITC1-16.

2. Anders HJ, Huber TB, Isermann B, et al. CKD in diabetes: diabetic kidney disease versus nondiabetic kidney disease. Nat Rev Nephrol 2018;14:361-77.

3. Zheng Y, Ley SH, Hu FB. Global Aetiology and Epidemiology of Type 2 Diabetes Mellitus and Its Complications. Nat Rev Endocrinol 2018;14:88-98.

4. Saaddine JB, Honeycutt AA, Narayan KMV, et al. Projection of diabetic retinopathy and other major eye diseases among people with diabetes mellitus: United States, 2005-2050. Arch Ophthalmol 2008;126:1740-7.

5. Mohamed Q, Gillies MC, Wong TY. Management of diabetic retinopathy: a systematic review. JAMA 2007;298:902-16.

6. Wong CW, Wong TY, Cheng C, et al. Kidney and Eye Diseases: Common Risk Factors, Etiological Mechanisms, and Pathways. Kidney Int 2014;85:1290-302.

7. Barber AJ. Diabetic Retinopathy: Recent Advances towards Understanding Neurodegeneration and Vision Loss. Sci China Life Sci 2015;58:541-9.

8. Carpineto P, Toto L, Aloia R, et al. Neuroretinal alterations in the early stages of diabetic retinopathy in patients with type 2 diabetes mellitus. Eye (Lond) 2016;30:673-9.

9. Pierro L, Iuliano L, Cicinelli MV, et al. Retinal Neurovascular Changes Appear Earlier in Type 2 Diabetic Patients. Eur J Ophthalmol 2017;27:346-51.

10. Srivastav K, Saxena S, Mahdi AA, et al. Increased Serum Urea and Creatinine Levels Correlate with Decreased Retinal Nerve Fibre Layer Thickness in Diabetic Retinopathy. Biomarkers 2015;20:470-3.

11. Lamb EJ, Stevens PE, Deeks JJ. What is the best glomerular filtration marker to identify people with chronic kidney disease most likely to have poor outcomes? BMJ 2015;350:g7667.

12. WHO Expert Consultation. Appropriate Body-mass Index for Asian Populations and Its Implications for Policy and Intervention Strategies. Lancet 2004;9403:157-63.

13. Li DY, Yin WJ, Yi YH, et al. Development and validation of a more accurate estimating equation for glomerular filtration rate in a Chinese population. Kidney Int 2019;95:636-46.

14. Thomas MC, Brownlee M, Susztak K, et al. Diabetic Kidney Disease. Nat Rev Dis Primers 2015;1:15018. 
15. Harding S, Greenwood R, Aldington S, et al. Grading and disease management in national screening for diabetic retinopathy in England and Wales. Diabet Med 2003;20:965-71.

16. Knight OJ, Girkin CA, Budenz DL, et al. Effect of race, age, and axial length on optic nerve head parameters and retinal nerve fiber layer thickness measured by Cirrus HDOCT. Arch Ophthalmol 2012;130:312-8.

Cite this article as: Gong X, Wang W, Li W, Jin L, Wang L, Meng J, Xiong K, Li Y, Han X, Liang X, Huang W. Association between renal function and retinal neurodegeneration in Chinese patients with type 2 diabetes mellitus. Ann Transl Med 2021;9(7):560. doi: 10.21037/atm-20-6957
17. Curcio CA, Allen KA. Topography of ganglion cells in human retina. J Comp Neurol 1990;300:5-25.

18. Cheung N, Mitchell P, Wong TY. Diabetic retinopathy. Lancet 2010;376:124-36.

19. Cunha-Vaz J, Faria de Abreu JR, Campos AJ. Early Breakdown of the Blood-retinal Barrier in Diabetes. Br J Ophthalmol 1975;59:649-56. 